\title{
Time-Resolved Spectroscopy of J1321: A Mysterious Polar with Deep Absorption Features
}

\author{
Taylor Hoyt ${ }^{* a}$ Colin Littlefield, ${ }^{b c}$ and Peter Garnavich ${ }^{b}$ \\ ${ }^{a}$ University of Texas at Austin, Austin, TX, United States \\ ${ }^{b}$ University of Notre Dame, Notre Dame, IN, United States \\ ${ }^{c}$ Wesleyan University, Middletown, CT, United States \\ E-mail: taylorjhoyteyahoo.com, clittlefealumni.nd.edu, \\ pgarnaviend.edu
}

\begin{abstract}
We present photometry and time-resolved spectroscopy of the polar MASTER OT J132104.04+560957.8 (J1321) taken at the Large Binocular Telescope (LBT) simultaneously with the LBC camera and MODS spectrograph in binocular mode. Our V-band photometry confirmed a primary and secondary maximum first identified by Littlefield et al. In the spectra we see unusually deep Balmer and helium absorption features for about 10-15\% of a 91 minute orbital period. The absorption lines first appear to the red of the emission peaks then shift bluewards, ultimately dominating the line profile at zero radial velocity. Towards the end of the absorption phase, weak absorption reappears to the blue of the emission lines. When the system is bright the spectra show a broadly-peaked continuum with a maximum around 500nm. We do not see absorption during the secondary bright red phase. Around the minima the continuum is blue and resembles a more typical polar spectrum with strong Balmer, neutral helium, and ionized helium emission lines. The Doppler tomograms of J1321 are fuzzy and blobby and resemble those of the asynchronous polar BY Cam. The highly variable width of the secondary maximum remains a mystery along with the lack of a discernible irradiated secondary in the tomograms. We use our observations to constrain the properties of the white dwarf's magnetic field as well as the accretion flow structure. We also discuss evidence of $\mathrm{J} 1321$ being in a prolonged low-mass-transfer state in all observations prior to 2007.
\end{abstract}

BASH 2015

18 - 20 October, 2015

The University of Texas at Austin, USA

\footnotetext{
* Speaker.
} 


\section{Observations, Analysis, and Modeling}

Long-slit spectra were taken using the MODS spectrograph on the Large Binocular Telescope with a $\sim 1 \AA$ wavelength resolution in the visible. The exposures were 120 s long with a 120 s dark time between them leading to a 0.04 phase resolution of J1321's 91 minute orbital period. The deep absorption lines, blobby doppler tomograms, and the hugely varying continuum (see Figure 1) point towards a high accretion rate in J1321 while the absence of distinct cyclotron lines during the bright phase indicates a low magnetic field strength $\sim 10 \mathrm{MG}$.

These two assumptions lead us to the conclusion that an extended accretion curtain is occulting the shock-front region near the surface of the WD for the duration of the absorption phase. To explain the absorption lines first appearing red of the emission then shifting bluewards towards velocity, we assume a particular viewing angle of the system such that the curtain begins eclipsing the hotspot right when the majority of the material in the curtain is traveling directly away from us at high velocities. This leads to the initial redshifted absorption lines. As the entire system continues to rotate, the curtain material then travels transverse to our line of sight and the absorption shifts bluewards towards the smaller orbital velocity. To confirm our assumptions about the accretion rate and field strength we can, from an approximate cyclotron emission theory, estimate the frequency spacing between subsequent cyclotron harmonics to be $\Delta \omega / \omega_{B} \sim$ $\left[1-(2 n+1) \frac{k_{b} T}{m c^{2}}\right]$ where $\mathrm{n}$ is the cyclotron harmonic number, $T$ is the emitting region temperature, $\omega_{b}$ the cyclotron resonance frequency, and $m c^{2}$ the rest mass of the electron. This behavior is modeled in [1] from the the-

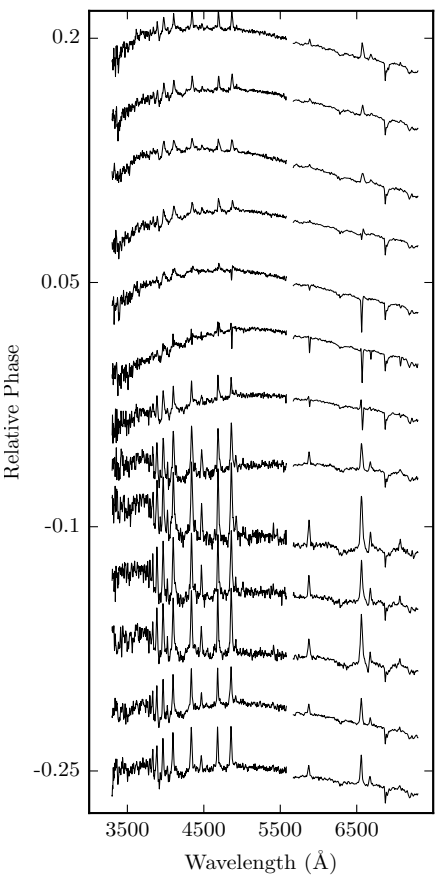

Figure 1: Sequence of spectra aligned vertically by relative phase. $\phi=0$ is assigned to the spectrum of deepest absorption. We can see the continuum shift from a typical polar spectrum to having one large hump centered around $500 \mathrm{~nm}$. Also note the interesting simultaneous absorption/emission line profile most clearly visible in $\mathrm{H}-\alpha$. The absorption lines first appear at redder wavelengths than the emission lines then shift bluewards. ory and techniques laid out in [2]. Thorough model fitting to the spectra will be found in a future publication (Littlefield et al. 2016, in prep).

\section{References}

[1] P.E. Barrett and G. Chanmugam, Cyclotron Lines in Accreting Magnetic White Dwarfs with an Application to VV Puppis, ApJ 298 (743-751).

[2] G. Chanmugam and G. A. Dulk, Polarized Radiation from Hot Plasmas and Applications to AM Herculis Binaries, ApJ 244 (569-578). 